\title{
Correlative nanoscopy: super resolved fluorescence and atomic force microscopy towards nanoscale manipulation and multimodal investigations.
}

\author{
Alberto Diaspro ${ }^{1,2}$, Jenu Chacko ${ }^{1,3}$, Francesca Cella Zanacchi ${ }^{1,2}$, Reiner Oropesa ${ }^{1,2}$, Silvia Dante $^{1}$, \\ Claudio Canale ${ }^{1}$ \\ 1. Istituto Italiano di Tecnologia, Nanoscopy and Nikon Imaging Center, Genova, Italy \\ 2. Department of Physics, University of Genoa, Genova, Italy. \\ 3. Biomedical Engineering Department, University of California, Irvine, United States.
}

Super resolved fluorescence microscopy combined with Atomic Force Microscopy allows to access different data sets and functionalities of investigated samples [1]. We named this hybrid approach coupling not specific force probing and fluorescence biochemical targeting - correlative nanoscopy. It opens opens new windows for approaching original questions to study behaviour of biological and materials science samples.

Here we mainly report about the positive synergy given by merging single molecule localization and targeted read-out super resolution methods with scanning force microscopy in a unique blend that allows nanoscale manipulation and multimodal investigations. More specifically, STED AFM and STORM AFM have proven to be very useful in its unique way of identifying species from an AFM image.

So far, the coupling between AFM and super resolved fluorescence optical microscopywas the first attempt to provide a chemical recognition to AFM by developing an integrated setup [2]. In the past, the main constraint of such an integration was given by low optical lateral resolution of the optical microscope, limited by diffraction at $\approx 250 \mathrm{~nm}$. Hence the two instruments worked at completely different spatial scales and molecular specific targeting allowed by fluorescence tagging could not be exploited at the nanoscale. The advent of optical approaches with unlimited spatial resolution offered a unique challenging opportunity for a correlative nanoscopy approach [3].

Now, AFM is a widely used technique for the reconstruction of biomaterials topography with a nanometric resolution. However, despite its high resolution, the nonspecific nature of the imaging provided by AFM does not allow to answer the biological queries focused on specific molecular targets within cellular and/or cytoskeletal compartments. Nowadays, fluorescence based super resolution techniques allow achieving precise localization of selected molecular species with a resolution far below the diffraction limit.

Within this scenario, one of the most advanced implementation of AFM is shown by the advantages of correlative approaches STED/AFM [2,4] and STORM/AFM [4,5], demonstrating a better comprehension of biological and biochemical questions and a strong integration of fluorescence imaging with the label free nature of the morphological information provided by AFM. We also show how the 3D imaging capabilities and multicolor STORM represents a powerful tool for a better overlay of the AFM and fluorescence information [4].

Moreover, the tip probing AFM approach opens a new window towards the possibility of mechanical nanomanipulation of cellular structures. Infact, the combined approach of AFM with the most advanced superresolution methods provides a golden opportunity for simultaneous nanomanipulation and imaging 
(with tens of nanometer resolution) of effects produced on specific molecule of interest in the cytoskeletal compartments of the cell $[4,6]$.

These techniques, based on correlative approaches, will smooth the way to a new generation of experiments able to couple topological information, local stiffness measurements and specific fluorescence imaging close to the molecular level.

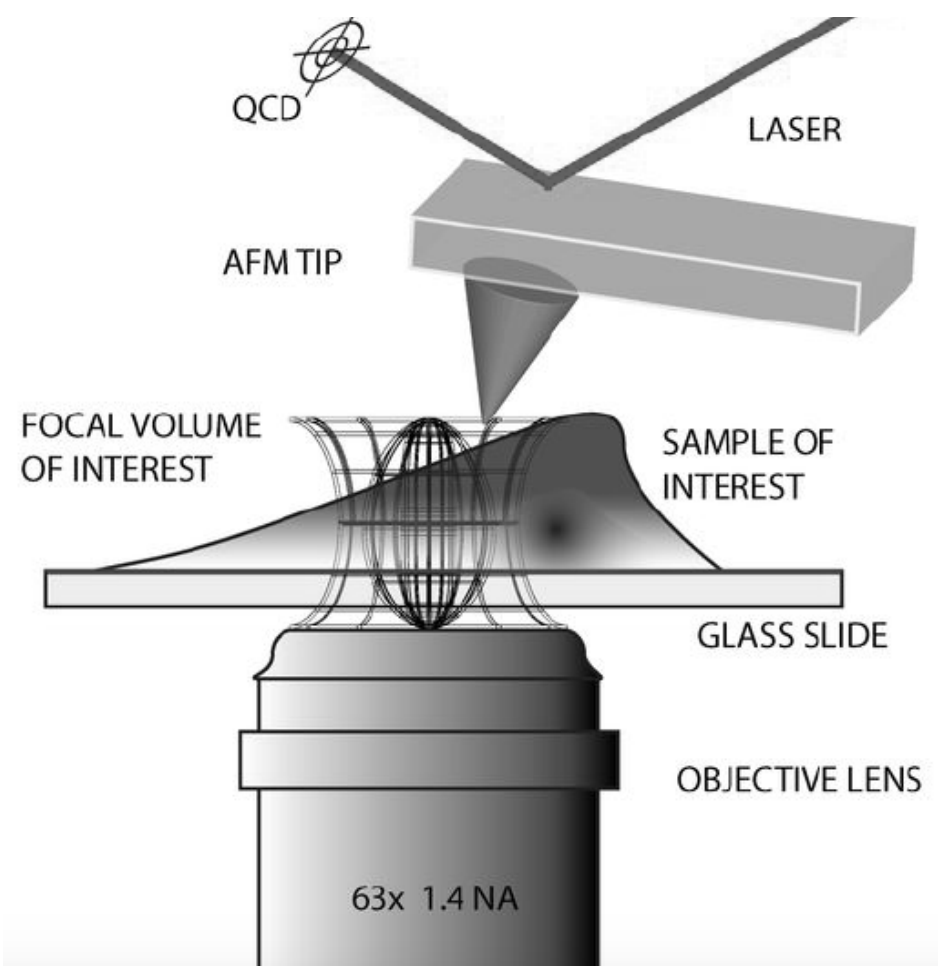

Figure 1. Schematic view of the STED-AFM coupling for correlative nanoscopy. Image credit J.Varghese Chacko.

References:

[1] C Smith, Nature 492 (2012) p. 293.

[2] B. Harke, J. V. Chacko, H. Haschke, C. Canale, A.Diaspro, Optical Nanoscopy 1 (2012) p.3.

[3] A. Diaspro, Il Nuovo Saggiatore 30 (2014) p.45.

[4] J. Varghese Chacko, F.Cella Zanacchi, and A. Diaspro. Cytoskeleton (Hoboken) 70 (2013) p.729

[5] A. Monserrate, S. Casado, and C. Flors, ChemPhysChem. 15 (2014) p. 647.

[6] J. Varghese Chacko, C. Canale, B. Harke, A. Diaspro, PLoS ONE 8 (2013) p.e66608. 